\title{
Infrared lock-in thermography for surface defect imaging in metals for industrial diagnosis
}

\author{
by M. Streza ${ }^{1,2}$, Y. Fedala ${ }^{2}$, J.-P. Roger ${ }^{3}$, G. Tessier ${ }^{3,4,5}$, C. Boué ${ }^{6}$ \\ ${ }^{1}$ NIR\&DIMT, 65-103 Donath Street, 400293 Cluj-Napoca, Romania \\ ${ }^{2}$ ESPCI, LPEM, 10 rue Vauquelin 75005 Paris, France \\ ${ }^{3}$ ESPCI, Institut Langevin, UMR CNRS 7587, 1 rue Jussieu 75005 Paris, France \\ ${ }^{4}$ CNRS UMR 8250, Neurophotonics Laboratory, Paris 75006, France \\ ${ }^{5}$ Université Paris 5 Descartes, 45 Rue des Saints Peres, Paris 75006, France \\ ${ }^{6}$ UPMC/ESPCI, LPEM, UMR CNRS 8213,10 rue Vauquelin 75005 Paris, France, \\ christine.boue@espci.fr
}

\begin{abstract}
Optical lock-in thermography is exploited for qualitative assessment of surface cracks in metallic samples. In order to identify and localize a surface defect, a special image processing of the recorded IR amplitude sequence is proposed. The obtained results demonstrate the potentiality of active lock-in thermography as a contactless measurement tool for localization of breaking cracks located into specific regions difficult to reach, and as a possible alternative to penetrant inspection in industrial processes.
\end{abstract}

\section{Introduction}

The requirements for non-destructive evaluation are driven by the need for low cost methods and instruments with great reliability, sensitivity and high operational speed, adapted to increasingly complex materials and structures [1]. In this context, infrared thermography seems attractive because of its 3D potential and noncontact character, which allows the control of most commonly encountered defects (impact damage, delamination, fatigue failure) $[2,3]$. The attention of the present study is focused on lock-in thermography for nondestructive inspection of various metallic components.

Thermography on highly reflecting objects is especially difficult, due to low emissivities and spurious IR reflections. Using lock-in thermography detection [4], the unwanted DC components (which depend on the emissivity of the materials and on the ambient temperature) can be filtered. The modulated component of the IR emission induced at the surface of the inspected component by the thermal wave is then extracted with an excellent contrast. As the thermal waves are disrupted by open fractures, which act as thermal barriers, the amplitude and phase of the modulated surface temperature bear the signature of their presence. The phase image processing could provide information concerning deep defects [5]. We will show that an analysis of amplitude images allows the selective detection of open cracks.

\section{Specimens and experimental procedure}

The investigated specimens with opened cracks of different depths, shapes and sizes, are low emissivity metallic samples. The tests were performed on specimens from the aeronautical and nuclear industries. An open vertical $30 \times 400 \mu \mathrm{m}^{2}$ fatigue crack in a highly reflecting $130 \times 27 \times 9 \mathrm{~mm}^{3}$ Inconel alloy metallic plate was used as model sample.

The experimental set up included a heat source (a diode pumped Ytterbium laser with 1.1 $\mu \mathrm{m}$ wavelength and $2 \mathrm{~W}$ power), a function generator, and the infrared camera. The IR camera (Jade III, FLIR) has an array (240x320 pixels) of InSb detectors which are sensitive in the $3-5 \mu \mathrm{m}$ wavelength range. The intensity-modulated optical radiation at a frequency $\mathrm{f}=0.2 \mathrm{~Hz}$ impinges on the inspected surface, close to the inspected defect. The signals delivered by the infrared camera and the reference frequency $f$ are sent to a lock-in detection module (FLIR R9902), which outputs the amplitude and phase images of the f-component to a PC.

\section{Results}

The lock-in detection module picks-up a series of thermal images and extracts a sinusoidal wave pattern at each pixel of the image. This lock-in thermography system analyses pixel by pixel the time dependence of the modulated response and displays the magnitude or phase of this modulation as an image. Finally, three images are obtained: the magnitude, the phase and the DC image (corresponding to the frequency $\mathrm{f}=0$ ).

The useful thermal signal has been recorded outside the area of laser impact. In order to be detected, the crack must be located inside the heat diffusion region, where the magnitude of thermal images have been analyzed. 
Typical images obtained for the Inconel alloy specimen are displayed in Figures (1) - (5).

Figure (1) shows the DC image. Because this specimen is highly reflective, the emissivity coefficient of the surface varies sharply in the presence of surface roughness, and consequently the resulting DC image is strongly disturbed. The defect is not clearly identified. However, by using the lock-in detection system, the image of the magnitude at the excitation frequency $f$ has been obtained. This image is free of artefacts induced by surface roughness, has a high signal-to-noise ratio, and one can clearly identify the laser impact area (Figure 2) and evaluate the extent of the heat diffusion region together with a disturbance in the profile of magnitude due to the crack (Figure 3).

In order to obtain a precise localization of the crack, signals in the laser impact area are filtered out. This was achieved by introducing a threshold factor that creates a mask for the magnitude image. The Laplace operator (the second derivative of the magnitude) is then applied to the image in order to highlight useful signal. In Figure 4, the image of the Laplacian is displayed outside the laser impact area.

Finally, by using an image thresholding and filtering technique, a binary image is obtained. Thermal barriers are clearly revealed: one can observe the vertical linear crack in addition to the edges of the sample (Figure 5).

The same procedure was applied to samples from the nuclear and aeronautical industries: cracks in turbine blades from terrestrial power generators or airplane engines were successfully identified, even in the case of irregular surface geometries.

We will present various applications illustrating the potential of lock-in thermography for reliable defect detection. The obtained results demonstrate the potentiality of active lock-in thermography as a contactless measurement tool for crack localization, specially in regions which are otherwise difficult to reach.

\section{REFERENCES}

[1] Avdelidis N.P., Gan T.H., Ibarra-Castanedo C., "Infrared thermography as a non-destructive tool for materials characterization and assessment". Proceedings of SPIE-The International Society for Optical Engineering, vol. 8013, paper 801313, DOI: 10.1117/12.887403, Orlando (United States), 2011.

[2] Legrandjacques L., Krapez J.-C., Lepoutre F., Balageas D., "Nothing but the cracks : a new kind of phototherma camera". Proceedings of $7^{\text {th }}$ European Conference on Nondestructive Testing, Copenhagen, 1998.

[3] Gruss C., Lepoutre F., Balageas D., " Nondestructive evaluation using a flying-spot camera”. Proceedings of $8^{\text {th }}$ Int. THERMO Conference, Budapest, 1993.

[4] Dillenz A., Zweschper T., Riegert G., Busse G., "Progress in phase angle thermography". Review of Scientific Instruments, vol. 74, pp. 417-419, 2003.

[5] Meola C., Carlomagno G. M., Squillace A., Vitiello A., "Non-destructive evaluation of aerospace materials with lock-in thermography". Engineering Failure Analysis, vol. 13, pp. 380-388, 2005

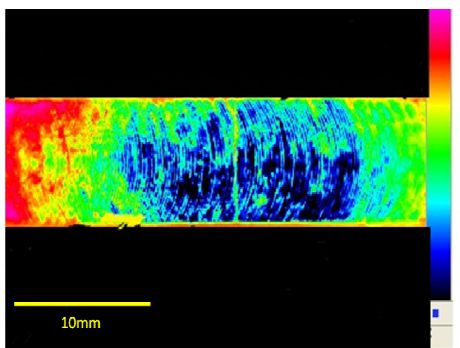

Fig.1. Continuous image.

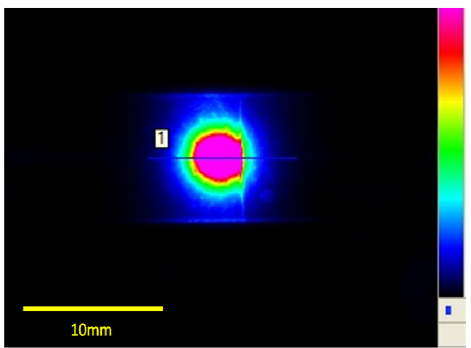

Fig.2. Normalized amplitude image.

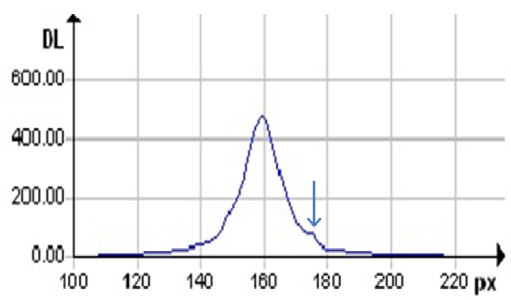

Fig.3. Profile of the amplitude.

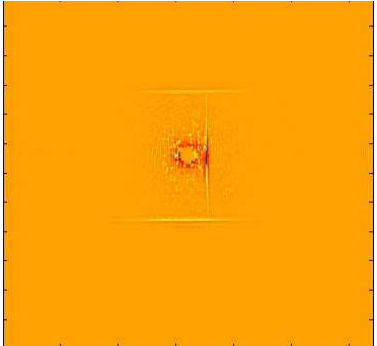

Fig.4. Laplace operator outside the laser impact area.

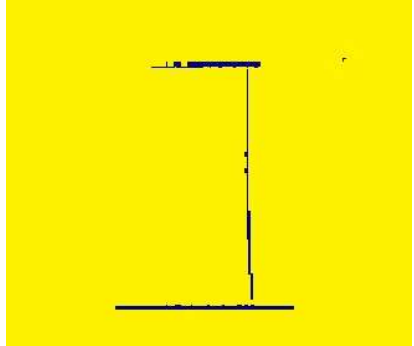

Fig.5. Enlarged view of the resulting binary image.

\section{ACKNOWLEDGMENTS}

This work was made possible thanks to the support of the "Pôle ASTech" and the "Pôle Nucléaire de Bourgogne", and has been financed by the Ville de Paris, France. 